\title{
Machine learning holographic mapping by neural network renormalization group
}

\author{
Hong-Ye Hu $\odot,{ }^{1}$ Shuo-Hui Li $\odot,{ }^{2,3}$ Lei Wang,,${ }^{2,4,5}$ and Yi-Zhuang You $\odot^{1, *}$ \\ ${ }^{1}$ Department of Physics, University of California at San Diego, La Jolla, California 92093, USA \\ ${ }^{2}$ Institute of Physics, Chinese Academy of Sciences, Beijing 100190, China \\ ${ }^{3}$ University of Chinese Academy of Sciences, Beijing 100049, China \\ ${ }^{4}$ CAS Center for Excellence in Topological Quantum Computation, University of Chinese Academy of Sciences, Beijing 100190, China \\ ${ }^{5}$ Songshan Lake Materials Laboratory, Dongguan, Guangdong 523808, China
}

(Received 28 February 2020; accepted 1 June 2020; published 19 June 2020)

\begin{abstract}
Exact holographic mapping (EHM) provides an explicit duality map between a conformal field theory (CFT) configuration and a massive field propagating on an emergent classical geometry. However, designing the optimal holographic mapping is challenging. Here we introduce the neural network renormalization group as a universal approach to design generic EHM for interacting field theories. Given a field theory action, we train a flow-based hierarchical deep generative neural network to reproduce the boundary field ensemble from uncorrelated bulk field fluctuations. In this way, the neural network develops the optimal renormalization-group transformations. Using the machine-designed EHM to map the CFT back to a bulk effective action, we determine the bulk geodesic distance from the residual mutual information. We have shown that the geometry measured in this way is the classical saddle-point geometry. We apply this approach to the complex $\phi^{4}$ theory in two-dimensional Euclidian space-time in its critical phase, and show that the emergent bulk geometry matches the three-dimensional hyperbolic geometry when geometric fluctuation is neglected.
\end{abstract}

DOI: 10.1103/PhysRevResearch.2.023369

\section{INTRODUCTION}

The holographic duality, also known as the anti-de Sitter space and conformal field theory correspondence (AdS-CFT) [1-4], is a duality between a CFT on a flat boundary and a gravitational theory in the AdS bulk with one higher dimension. It is intrinsically related to the renormalization-group (RG) flow [5-11] of the boundary quantum field theory, since the dilation transformation, as a part of the conformal group, naturally corresponds to the coarse-graining procedure in the RG flow. The extra dimension emergent in the holographic bulk can be interpreted as the RG scale. In the traditional real-space RG [12], the coarse-graining procedure decimates irrelevant degrees of freedom along the RG flow, therefore the RG transformation is irreversible due to information loss. However, if the decimated degrees of freedom are collected and hosted in the bulk, the RG transformation becomes a bijective map between the degrees of freedom on the CFT boundary and the degrees of freedom in the AdS bulk. Such mappings, generated by information-preserving RG transforms, are called exact holographic mappings (EHMs) [13-15], which were first formulated for free-fermion CFT. Under the EHM, the boundary features of a quantum field

\footnotetext{
*yzyou@physics.ucsd.edu

Published by the American Physical Society under the terms of the Creative Commons Attribution 4.0 International license. Further distribution of this work must maintain attribution to the author(s) and the published article's title, journal citation, and DOI.
}

theory of different scales are mapped to different depths in the bulk, and vice versa. The field variable deep in the bulk represents the overall or infrared (IR) features, while the variable close to the boundary controls the detailed or ultraviolet (UV) features. Such a hierarchical arrangement of information is often observed in deep neural networks, particularly in convolutional neural networks (CNNs) [16]. The similarity between renormalization group and deep learning has been discussed in several works [17-22]. With the development of machine learning in quantum many-body physics [23-28], deep learning techniques have also been employed to construct the optimal RG transformations [29,30] and to uncover the holographic geometry [31-34]. In this work, we further explore the possibility of designing the EHM for interacting quantum field theories using deep learning approaches. By training a flow-based hierarchical generative model $[35,36]$ to generate field configurations following the probability distribution specified by the field theory action, the model converges to the optimal EHM with an emergent bulk gravitational description, where neural network parameters and latent variables correspond respectively to the gravity (geometry) and matter degrees of freedom in the holographic bulk. The learned holographic mapping is useful for both sampling and inference tasks. In the sampling task, the generative model is used to propose efficient global updates for boundary field configurations, which helps to boost the Monte Carlo simulation efficiency of the CFT. In the inference task, the boundary field theory is pulled back to an effective theory of the bulk field, which enables us to probe the emergent dual geometry (on the classical level) by measuring the mutual information in the bulk field. 

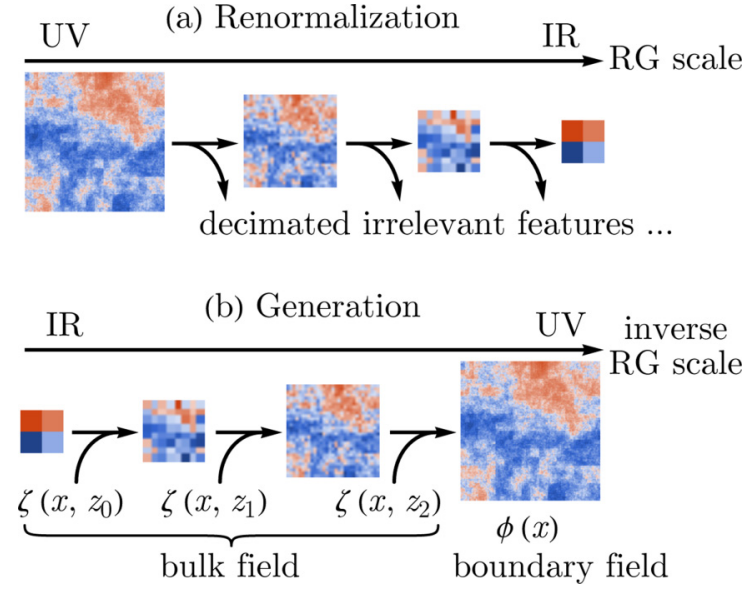

FIG. 1. Relation between (a) RG and (b) generative model. The inverse RG can be viewed as a generative model that generates the ensemble of field configurations from random sources. The random sources are supplied at different RG scales (coordinated by $z$ ), which can be viewed as a field $\zeta(x, z)$ living in the holographic bulk with one more dimension. The original field $\phi(x)$ is generated on the holographic boundary.

\section{RENORMALIZATION GROUP AND GENERATIVE MODEL}

Renormalization group ( $R G)$ plays a central role in the study of quantum field theory (QFT) and many-body physics. The RG transformation progressively coarse-grains the field configuration to extract relevant features. The coarse-graining rules (or the RG schemes) are generally model dependent and require human design. Take the real-space RG [12] for example: for a ferromagnetic Ising model, the RG rule should progressively extract the uniform spin components as the most relevant feature; however, for an antiferromagnetic Ising model, the staggered spin components should be extracted instead; if the spin couplings are randomly distributed on the lattice, the RG rule can be more complicated. When it comes to the momentum-space RG [37], the rule becomes to renormalize the low-energy degrees of freedom by integrating out the high-energy degrees of freedom. What is the general design principle behind all these seemly different RG schemes? Can a machine learn to design the optimal RG scheme based on the model action?

With these questions in mind, we take a closer look at the $\mathrm{RG}$ procedure in a lattice field theory setting. In the traditional RG approach, the RG transformation is invertible due to information loss at each RG step when the irrelevant features are decimated, as illustrated in Fig. 1(a). However, if the decimated features are kept at each RG scale, the RG transformation can be inverted. Under the inverse RG flow, the decimated degrees of freedom $\zeta(x, z)$ are supplied to each layer (step) of the inverse RG transformation such that the field configuration $\phi(x)$ can be regenerated, as shown in Fig. 1(b). Here we assume that the $\phi(x)$ field is defined in a flat Euclidean space-time coordinated by $x=\left(x_{1}, x_{2}, \ldots\right) \in \mathbb{R}^{d}$, then $\zeta(x, z)$ will live on a manifold with one higher dimension, and the extra dimension $z$ corresponds to the RG scale. Given its close analogy to the holographic duality, we may view $\zeta(x, z)$ as the field in the holographic bulk and $\phi(x)$ as the field on the holographic boundary. The inverse RG can be considered as a deep generative model $G$, which organizes the bulk field $\zeta(x, z)$ to generate the boundary field $\phi(x)$,

$$
\phi(x)=G[\zeta(x, z)] .
$$

The renormalization $G^{-1}$ and generation $G$ procedures are thus unified as the forward and backward maps of a bijective (invertible) map between the boundary and the bulk, known as the EHM [13,14].

At first glance, such an information-preserving RG does not seem to have much practical use, because $\zeta(x, z)$ is simply a rewriting of $\phi(x)$, which does not seem to reduce the degrees of freedom. However, since the bulk field $\zeta(x, z)$ represents the irrelevant feature to be decimated under RG, it should look like independent random noise, which contains a minimal amount of information. So instead of memorizing the bulk field configuration $\zeta(x, z)$ at each $\mathrm{RG}$ scale for reconstruction purposes, we can simply sample $\zeta(x, z)$ from uncorrelated (or weakly correlated) random source and serve them to the inverse RG transformation. Suppose the bulk field $\zeta(x, z)$ is drawn from a prior distribution $P_{\text {prior }}[\zeta]$, the transformation $\phi=G[\zeta]$ will deform the prior distribution to a posterior distribution $P_{\text {post }}[\phi]$ for the boundary field $\phi(x)$,

$$
P_{\text {post }}[\phi]=P_{\text {prior }}[\zeta]\left|\operatorname{det}\left(\frac{\delta G[\zeta]}{\delta \zeta}\right)\right|^{-1},
$$

where $\left|\operatorname{det}\left(\delta_{\zeta} G\right)\right|^{-1}$ is the Jacobian determinant of transformation. In such a manner, the objective of the inverse RG is not to reconstruct a particular original field configuration, but to generate an ensemble of field configurations $\phi(x)$, whose probability distribution $P_{\text {post }}[\phi]$ should better match the Boltzmann distribution,

$$
P_{\text {target }}[\phi]=e^{-S_{\mathrm{QFT}}[\phi]} / Z_{\mathrm{QFT}},
$$

specified by the action functional $S_{\mathrm{QFT}}[\phi(x)]$ of the boundary field theory, where $Z_{\mathrm{QFT}}=\sum_{[\phi]} e^{-S_{\mathrm{QFT}}[\phi]}$ denotes the partition function.

This setup provides us a theoretical framework to discuss the designing principles of a good RG scheme. We propose two objectives for a good RG scheme (or EHM): the RG transformation should aim at decimating irrelevant features and preserving relevant features, and the inverse RG must aim at generating field configurations matching the target field theory distribution $P_{\text {target }}[\phi]$ in Eq. (3). An information theoretic criterion for "irrelevant" features is that they should have minimal mutual information, so the prior distribution $P_{\text {prior }}[\zeta]$ should be chosen to minimize the mutual information between bulk fields at different points, i.e., $\min I\left(\zeta(x, z): \zeta\left(x^{\prime}, z^{\prime}\right)\right)$. We refer to this designing principle as the minimal bulk mutual information (minBMI) principle, which is a general information theoretic principle behind different RG schemes and is independent of the notion of field pattern or energy scale. The close relation between RG and deep learning has been discussed in several early works [18,20,21]. However, as pointed out in Refs. [30,38], the hierarchical architecture itself cannot guarantee the emergence of RG transformation in a deep neural network. Additional information theoretic principles must be imposed to guide the learning. In light of this observation, Refs. [30,38] proposed the maximal 
real-space mutual information (maxRSMI) principle, which aims at maximizing the mutual information between the coarse-grained field and the fine-grained field in the surrounding environment. Our minBMI principle is consistent with and is more general than the maxRSMI principle (see Appendix A for a detailed discussion about the relation between these two principles).

In the simplest setting, we can hard code the minBMI principle by assigning the prior distribution to the uncorrelated Gaussian distribution,

$$
P_{\text {prior }}[\zeta]=\mathcal{N}[\zeta ; 0,1] \propto e^{-\|\zeta\|^{2}},
$$

where $\|\zeta\|^{2}=\sum_{x, z}|\zeta(x, z)|^{2}$. Hence the mutual information vanishes for every pair of points in the holographic bulk. Given the prior distribution, the problem of finding the optimal EHM boils down to training the optimal generative model $G$ to minimize the Kullback-Leibler (KL) divergence between the posterior distribution $P_{\text {post }}[\phi]$ in Eq. (2) and the target distribution $P_{\text {target }}[\phi]$ in Eq. (3), i.e., $\min \mathcal{L}$ with

$$
\begin{aligned}
\mathcal{L} & =\operatorname{KL}\left(P_{\text {post }}[\phi] \| P_{\text {target }}[\phi]\right) \\
& =\underset{\zeta \sim P_{\text {prior }}}{\mathbb{E}} S_{\mathrm{QFT}}[G[\zeta]]+\ln P_{\text {prior }}[\zeta]-\ln \operatorname{det}\left(\frac{\delta G[\zeta]}{\delta \zeta}\right),
\end{aligned}
$$

where $\mathbb{E}_{\zeta \sim P_{\text {prior }}}$ denotes the average over the ensemble of $\zeta$ drawn from the prior distribution. This fits perfectly to the framework of flow-based generative models $[35,36]$ in machine learning, which can be trained efficiently thanks to its tractable and differentiable posterior likelihood. We model the bijective map $G$ by a neural network (to be detailed later) with trainable network parameters. We initiate the sampling from the bulk $\zeta \sim P_{\text {prior }}$ and push the bulk field to the boundary by $\phi=G[\zeta]$, collecting the logarithm of the Jacobian determinant along the way. Given the action $S_{\mathrm{QFT}}[\phi]$, we can evaluate the loss function $\mathcal{L}$ in Eq. (5) and back propagate its gradient with respect to the network parameters. We then update the network parameters by stochastic gradient descent. We iterate the above steps to train the neural network. In this way, simply by presenting the QFT action $S_{\mathrm{QFT}}$ to the machine, the machine learns to design the optimal RG transformation $G$ by continually probing $S_{\mathrm{QFT}}$ with various machine-generated field configurations. Thus our algorithm may be called the neural network renormalization group (neural RG) [29], which can be implemented by using deep learning platforms such as TENSORFLOW [39].

\section{HOLOGRAPHIC DUALITY AND CLASSICAL APPROXIMATION}

We would like to provide an alternative interpretation of the loss function $\mathcal{L}$ in Eq. (5) in the context of holographic duality, which will deepen our understanding of the capabilities and limitations of our approach. Suppose we can sample the boundary field configuration $\phi(x)$ from the target distribution $P_{\text {target }}[\phi]$ and map $\phi(x)$ to the bulk by applying the EHM along the RG direction $\zeta=G^{-1}[\phi]$, the obtained bulk field $\zeta(x, z)$ will follow the distribution

$$
\begin{aligned}
P_{\text {bulk }}[\zeta] & =P_{\text {target }}[\phi] \operatorname{det}\left(\delta_{\phi} G^{-1}[\phi]\right)^{-1} \\
& =Z_{\mathrm{QFT}}^{-1} e^{-S_{\mathrm{QFT}}[G[\zeta]]} \operatorname{det}\left(\delta_{\zeta} G\right),
\end{aligned}
$$

where we have used Eq. (3) to express $P_{\text {target }}[\phi]$ in terms of $\zeta$. The normalization of the bulk field probability distribution $\sum_{[\zeta]} P_{\text {bulk }}[\zeta]=1$ further implies that the QFT partition function $Z_{\mathrm{QFT}}$, which was originally defined on the holographic boundary, can now be written in terms of the bulk field $\zeta$ as well

$$
Z_{\mathrm{QFT}}=\sum_{[\zeta]} e^{-S_{\mathrm{QFT}}[G[\zeta]]+\ln \operatorname{det}\left(\delta_{\zeta} G\right)} .
$$

Note that $Z_{\mathrm{QFT}}$ is by definition independent of $G$, so we are allowed to sum over all possible $G$ on both sides of Eq. (7), which establishes a duality between the following two partition functions:

$$
Z_{\mathrm{QFT}}=\sum_{[\phi]} e^{-S_{\mathrm{QFF}}[\phi]} \leftrightarrow Z_{\mathrm{grav}}=\sum_{[\zeta, G]} e^{-S_{\mathrm{grav}}[\zeta, G]}
$$

with the bulk theory $S_{\text {grav }}$ given by

$$
S_{\mathrm{grav}}[\zeta, G]=S_{\mathrm{QFT}}[G[\zeta]]-\ln \operatorname{det}\left(\delta_{\zeta} G\right) .
$$

By "duality" we mean that $Z_{\mathrm{QFT}}$ and $Z_{\text {grav }}$ only differ by a proportionality constant (because $Z_{\mathrm{grav}}=\sum_{[G]} Z_{\mathrm{QFT}}$ ), so they are equivalent descriptions of the same physics theory. $S_{\text {grav }}[\zeta, G]$ describes how the bulk variables $\zeta$ (matter field) and the neural network $G$ (geometry) would fluctuate and interact with each other, which resembles a "quantum gravity" theory in the holographic bulk. The bulk has more degrees of freedom than the boundary, because there can be many different choices of $\zeta$ and $G$ that leads to the same boundary field configuration $\phi=G[\zeta]$. This is a gauge redundancy in the bulk theory, which covers the diffeomorphism invariance as well as the interchangeable role between matter and spacetime geometry in a gravity theory. At this level, the bulk theory looks intrinsically nonlocal and the geometry can fluctuate strongly.

However, it is usually more desired to work with quantum gravity theories with a classical limit, which describe weak fluctuations (matter fields and gravitons) around a classical geometry. Although not every CFT admits a classical gravity dual, we still attempt to find the classical approximation of the dual quantum gravity theory, neglecting the fluctuation of $G$. Aiming at a classical geometry, we look for the optimal $G$ that maximizes its marginal probability $P_{\mathrm{EHM}}[G]=$ $Z_{\text {grav }}^{-1} \sum_{[\zeta]} e^{\left.-S_{\text {grav }} \zeta, G\right]}$ with the bulk-matter field $\zeta$ traced out. This optimization problem seems trivial because, according to Eq. (7), $P_{\mathrm{EHM}}[G]=Z_{\mathrm{QFT}} / Z_{\mathrm{grav}}$ is independent of $G$. It is understandable that any choice of $G$ is equally likely if we have no preference on the prior distribution $P_{\text {prior }}[\zeta]$ of the bulk-matter field, because there is a trade-off between $G$ and $P_{\text {prior }}$ that one can always adjust $P_{\text {prior }}$ to compensate the change in $G$. Such a trade-off behavior is fundamentally required by the gauge redundancy in the bulk gravity theory. To fix the gauge, we evoke the minBMI principle to bias the bulk-matter field toward independent random noise, such that the classical solution of $G$ will look like a RG transformation, in line with our expectation for a holographic mapping. Choosing a minBMI prior distribution such as Eq. (4) and replacing $\operatorname{det}\left(\delta_{\zeta} G\right)$ in Eq. (9) by $P_{\text {prior }} / P_{\text {post }}, P_{\text {EHM }}[G]$ can be 
cast into

$$
P_{\mathrm{EHM}}[G]=\frac{Z_{\mathrm{QFT}}}{Z_{\text {grav }}} \underset{\zeta \sim P_{\text {prior }}}{\mathbb{E}} \frac{P_{\text {target }}[G[\zeta]]}{P_{\text {post }}[G[\zeta]]} \geqslant \frac{Z_{\mathrm{QFT}}}{Z_{\text {grav }}} e^{-\mathcal{L}},
$$

which is bounded by $e^{-\mathcal{L}}$ from below, with $\mathcal{L}$ being the $\mathrm{KL}$ divergence between $P_{\text {post }}$ and $P_{\text {target }}$ as defined in Eq. (5). Therefore the objective of maximizing $P_{\mathrm{EHM}}[G]$ can be approximately replaced by minimizing the loss function $\mathcal{L}$, which is no longer a trivial optimization problem. From this perspective, the loss function $\mathcal{L}$ can be approximately interpreted as the action (negative log-likelihood) for the holographic bulk geometry associated with the EHM G. Minimizing the loss function corresponds to finding the classical saddle-point solution of the bulk geometry. We will build a flow-based generative model to parametrize $G$ and train the neural network by using deep-learning approaches. The fluctuation of neural network parameters in the learning dynamics reflects (at least partially) the gravitational fluctuation in the holographic bulk.

At the classical saddle point $G_{*}=\operatorname{argmin}_{G} \mathcal{L}$, we may extract an effective theory for the bulk-matter field:

$$
\begin{aligned}
S_{\mathrm{eff}}[\zeta] & \equiv S_{\mathrm{grav}}\left[\zeta, G_{*}\right] \\
& =\|\zeta\|^{2}+\ln P_{\mathrm{post}}\left[G_{*}[\zeta]\right]-\ln P_{\text {target }}\left[G_{*}[\zeta]\right] .
\end{aligned}
$$

As the $\mathrm{KL}$ divergence $\mathcal{L}=\mathrm{KL}\left(P_{\text {post }} \| P_{\text {target }}\right)$ is minimized after training, we expect $P_{\text {post }}$ and $P_{\text {target }}$ to be similar, such that their $\log$-likelihood difference $\ln P_{\text {post }}-\ln P_{\text {target }}$ will be small, so the effective theory $S_{\text {eff }}[\zeta]$ will be dominated by the first term $\|\zeta\|^{2}$ in Eq. (11), implying that the bulk field $\zeta$ will be massive. The small log-likelihood difference further provides kinetic terms (and interactions) for the bulk field $\zeta$, allowing it to propagate on a classical background that is implicitly specified by $G_{*}$. In this way, the bulk field will be correlated in general. Even though one of our objectives is to minimize the bulk mutual information as much as possible, the learned EHM typically cannot resolve all correlations in the original QFT, so the residual correlations will be left in the bulk field $\zeta$ as described by the log-likelihood difference in Eq. (11). The mismatch between $P_{\text {post }}$ and $P_{\text {target }}$ may arise for several reasons: first, limited by the design of the neural network, the generative model $G$ may not be expressive enough to precisely deform the prior distribution to the target distribution; second, even if $G$ has sufficient representation power, the training may not be able to converge to the global minimum; finally and perhaps the most fundamental reason is not every QFT has a classical gravitational dual; the bulk theory should be quantum gravity in general. Taking the classical approximation and ignoring the gravitational fluctuation leads to the unresolvable correlation and interaction for the matter field $\zeta$ that has to be kept in the bulk.

Nevertheless, our framework could in principle include fluctuations of $G$ by falling back to $Z_{\text {grav }}$ in Eq. (8). We can either model the marginal distribution $P_{\mathrm{EHM}}[G]$ by techniques like graph generative models, or directly analyze the gravitational fluctuations by observing the fluctuations of neural network parameters in the learning dynamics as mentioned below Eq. (10). We will leave these ideas for future exploration. In the following, we will use a concrete example, a two-dimensional (2D) compact boson CFT on a lattice, to illustrate our approach of learning the EHM as a generative model and to demonstrate its applications in both the sampling and the inference tasks.

\section{APPLICATION TO COMPLEX $\phi^{4}$ MODEL}

We consider a lattice field theory defined on a 2D square lattice, described by the Euclidean action

$$
S_{\mathrm{QFT}}[\phi]=-t \sum_{\langle i j\rangle} \phi_{i}^{*} \phi_{j}+\sum_{i}\left(\mu\left|\phi_{i}\right|^{2}+\lambda\left|\phi_{i}\right|^{4}\right),
$$

where $\phi_{i} \in \mathbb{C}$ is a complex scalar field defined on each site $i$ of a square lattice and $\langle i j\rangle$ denotes the summation over all nearest-neighbor sites. The model has global U(1) symmetry under which the field rotates by $\phi_{i} \rightarrow e^{\mathrm{i} \varphi} \phi_{i}$ on every site. We choose $\mu=-200+2 t$ and $\lambda=25$ to create a deep Mexican hat potential that basically pins the complex field on a circle $\phi_{i}=\sqrt{\rho} e^{\mathrm{i} \theta_{i}}$ of radius $\sqrt{\rho}=2$. In this way, the field theory falls back to the $X Y$ model $S_{\mathrm{QFT}}=-\frac{1}{T} \sum_{\langle i j\rangle} \cos \left(\theta_{i}-\theta_{j}\right)$ with an effective temperature $T=(\rho t)^{-1}$. By tuning the temperature $T$, the model exhibits two phases: the low- $T$ algebraic liquid phase with a powerlaw correlation $\left\langle\phi_{i}^{*} \phi_{j}\right\rangle \sim\left|x_{i}-x_{j}\right|^{-\alpha}$ and the high- $T$ disordered phase with a short-range correlation. The two phases are separated by the Kosterlitz-Thouless (KT) transition. Several recent works [40-43] have focused on applying machine learning methods to identify phase transitions or topological defects (vortices). Our purpose is different here: we stay in the algebraic liquid phase described by a Luttinger liquid CFT and seek to develop the optimal holographic mapping for the CFT.

We design the generative model $G$ as a bijective deep neural network following the architecture of the neural network renormalization group (neural-RG) proposed by Ref. [29]. Its structure resembles the MERA network [44] as depicted in Fig. 2(a). Each RG step contains a layer of disentangler blocks (like a CNN convolutional layer) to resolve local correlations, and a layer of decimator blocks (like a $\mathrm{CNN}$ pooling layer) to separate the renormalized and decimated variables. Given that the space-time dimension is two on the boundary, we can overlay decimators on top of disentanglers in an interweaving manner as shown in Fig. 2(b). Both the disentangler and the decimator are made of three bijective layers: a linear scaling layer, an orthogonal transformation layer, and an invertible nonlinear activation layer, as arranged in Fig. 3 (see Appendix B for more details). They are designed to be invertible, nonlinear, and U(1)-symmetric transformations, which are used to model generic RG transformations for the complex $\phi^{4}$ model. The bijector parameters are subject to training (training process and number of parameters are specified in Appendix C). The Jacobian matrix of these transformations are calculable. After each decimator, only one renormalized variable flows to the next RG layer, and the other three decimated variables are positioned into the bulk as little crosses as shown in Figs. 2(a) and 2(b). The entire network constitutes an EHM between the original boundary field $\phi(x)$ and the dual field $\zeta(x, z)$ in the holographic bulk.

We start with a $32 \times 32$ square lattice as the holographic boundary and build up the neural-RG network. The network will have five layers in total. Since the boundary field theory has a global U(1) symmetry, the bijectors in the neural network are designed to respect the U(1) symmetry (see Fig. 3), 
(a)

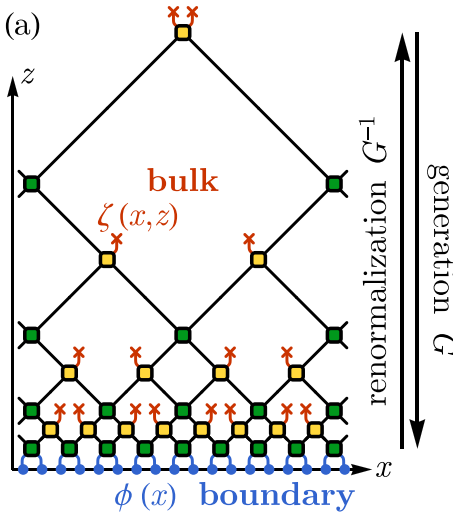

(c)

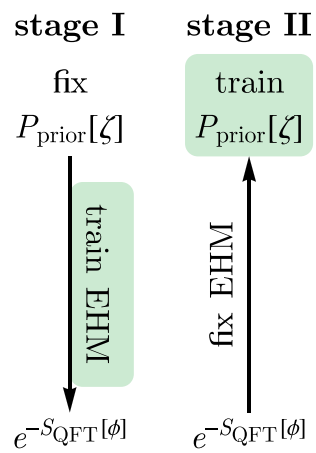

(b)

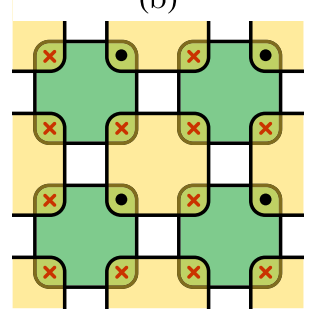

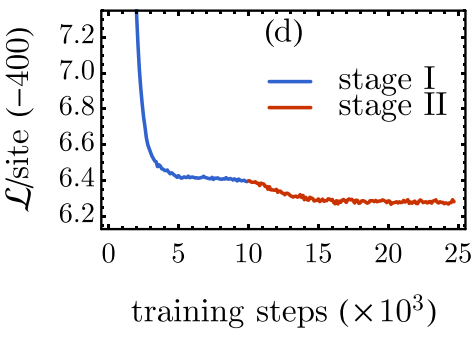

FIG. 2. (a) Side view of the neural-RG network. $x$ is the spatial dimension(s) and $z$ corresponds to the RG scale. There are two types of blocks: disentanglers (dark green) and decimators (light yellow). The network forms an EHM between the boundary variables (blue dots) and the bulk variables (red crosses). (b) Top view of one RG layer in the network. Disentanglers and decimators interweave in the space-time (taking two-dimensional space-time, for example). Each decimator pushes the coarse-grained variable (black dot) to the higher layer and leaves the decimated variables (red crosses) in the holographic bulk. (c) The training contains two stages. In the first stage, we fix the prior distribution $P[\zeta]$ to be uncorrelated Gaussian and train the EHM $G$ to bring it to the Boltzmann distribution of the CFT. In the second stage, we learn the prior distribution with the trained EHM held fixed. (d) The behavior of the loss function $\mathcal{L}$ in the two training stages.

such that the bulk field also preserves the U(1) symmetry. The training will be divided into two stages, as pictured in Fig. 2(c). In the training stage I, we fix the prior distribution in Eq. (4) and train the network parameters in the generative model $G$ to minimize the loss function $\mathcal{L}$. The training method is outlined below Eq. (5). The loss function $\mathcal{L}$ decays with training steps, whose typical behavior is shown in Fig. 2(d). We will discuss the stage II training later.

We perform the stage I training for several neural networks at different temperatures $T$ separately, i.e., we use $S_{\mathrm{QFT}}[\phi]$ of different parameters to train different neural networks. After training, each neural network can generate configurations of the boundary field $\phi$ from the bulk uncorrelated Gaussian field $\zeta$ efficiently. To test how well these generative models work, we measured the order parameter $\langle\phi\rangle$ and the correlation function $\left\langle\phi_{i}^{*} \phi_{j}\right\rangle$ using the field configurations generated by the neural network. Although the order parameter $\langle\phi\rangle$ is expected to vanish in the thermodynamic limit, for our finitesize system, it is not vanishing and can exhibit a crossover around the KT transition, as shown in Fig. 4(a). The crossover

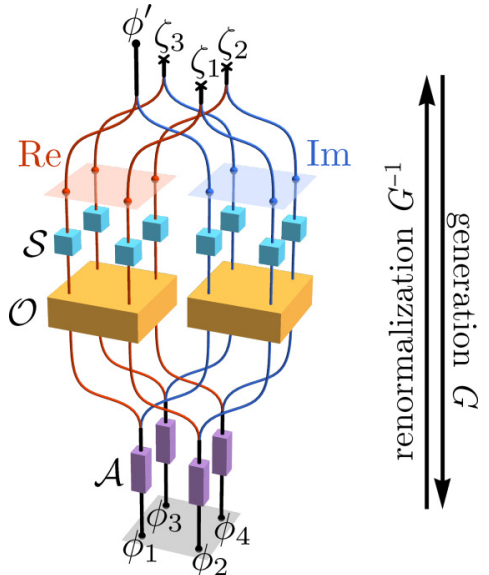

FIG. 3. Neural network architecture within a decimator block (the disentangler block shares the same architecture). Starting from the renormalized variable $\phi^{\prime}$ and the bulk noise $\zeta_{1}, \zeta_{2}, \zeta_{3}$ as complex variables, the $\operatorname{Re}$ and Im channels are first separated, then $\mathcal{S}$ applies the scaling separately to the four variables within each channel and $\mathcal{O}$ implements the $\mathrm{O}(4)$ transformation that mixes the four variables together. $\mathcal{S}$ and $\mathcal{O}$ are identical for Re and Im channels to preserve the $\mathrm{U}(1)$ symmetry. Then the channels merge into complex variables followed by element-wise nonlinear activation describe by an invertible U(1)-symmetric map $\phi_{i} \mapsto\left(\phi_{i} /\left|\phi_{i}\right|\right) \sinh \left|\phi_{i}\right|$.
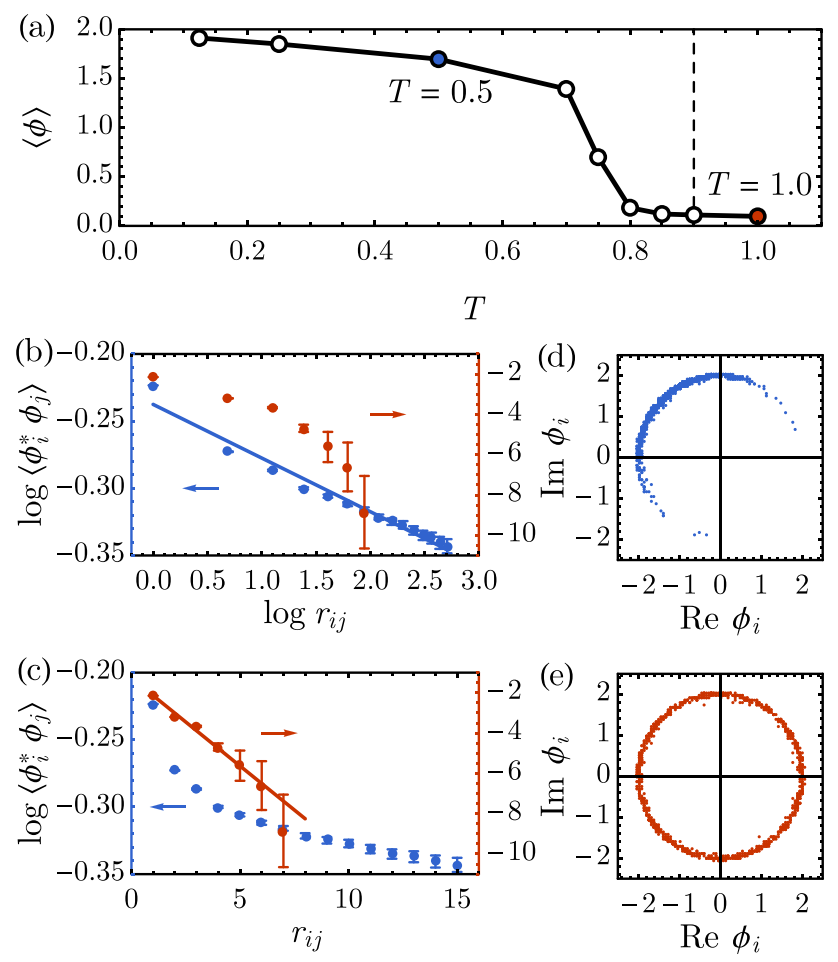

FIG. 4. Performance of the trained EHM for the complex $\phi^{4}$ theory. (a) Order parameter $\langle\phi\rangle$ vs temperature $T$. Different models are trained separately at different temperature. For finite-sized system, $\langle\phi\rangle$ crosses over to zero around the KT transition. Correlation function $\left\langle\phi_{i}^{*} \phi_{j}\right\rangle$ scaling in log-log plot (b) and log-linear plot (c). Distribution of $\phi_{i}$ in a single sample generated by the neural network trained in (d) the algebraic liquid phase and (e) the disordered phase. 

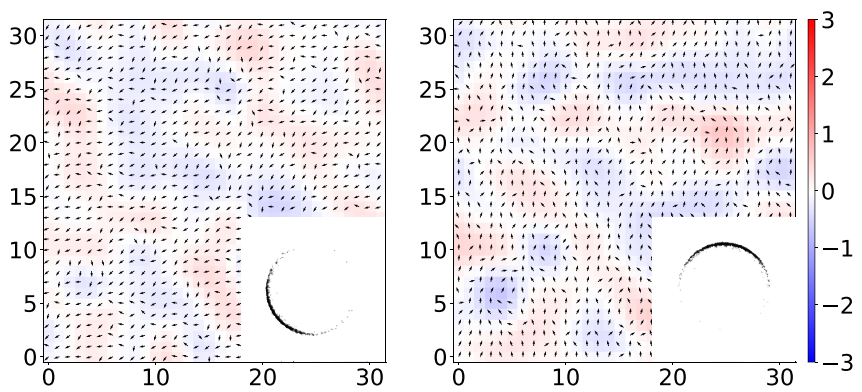

FIG. 5. The boundary field configuration $\phi$ before (left) and after (right) a local update in the most IR layer of the bulk field $\zeta$. The complex field $\phi_{i}$ is represented by the small arrow on each site. The background color represents the vorticity. The inset shows the distribution of $\phi_{i}$ in the complex plane.

temperature $T \simeq 0.9$ agrees with the previous Monte Carlo study [45-47] of the KT transition temperature $T_{\mathrm{KT}}=0.8929$ in the two-dimensional $X Y$ model. We measure the correlation function $\left\langle\phi_{i}^{*} \phi_{j}\right\rangle$ at two different temperatures: one at $T=0.5$ in the algebraic liquid phase, one at $T=1.0$ in the disordered phase. We plot the two-point function $\left\langle\phi_{i}^{*} \phi_{j}\right\rangle$ as a function of the Euclidean distance $r_{i j} \equiv\left|x_{i}-x_{j}\right|$ (on the square lattice) on both a log-log scale as in Fig. 4(b) and on a log-linear scale as in Fig. 4(c). The comparison shows that the correlation function in the algebraic liquid (or the disordered) phase fits better to the power-law (or the exponential) decay. Figure 4(d) shows the statistics of $\phi_{i}$ in one sample generated by the machine trained in the algebraic liquid phase. It exhibits the "spontaneous symmetry breaking" behavior due to the finitesize effect, although accumulating over multiple samples will restore the U(1) symmetry. However, the similar plot Fig. 4(e) in the disordered phase respects $U(1)$ symmetry in every single sample. Based on these tests, we conclude that the neural network has learned to generate field configurations $\phi(x)$ that reproduce the correct physics of the complex $\phi^{4}$ model. The trained generative model $G$ maps an almost uncorrelated bulk field $\zeta$ to a correlated boundary field $\phi$, and vice versa, therefore $G$ provides a good EHM for the $\phi^{4}$ theory.

The learned EHM can be useful in both the backward and forward directions. The backward mapping from bulk to boundary provides efficient sampling of the CFT configurations, which can be used to boost the Monte Carlo simulation of the CFT. The forward mapping from boundary to bulk enables direct inference of bulk field configurations, allowing us to study the bulk effective theory and to probe the bulk geometry. Let us first discuss the sampling task. The EHM establishes a mapping between the massive bulk field $\zeta$ and the massless boundary field $\phi$. The bulk field admits efficient sampling in terms of local update, because the it is uncorrelated (or short-range correlated). Local updates in the bulk gets maps to global updates on the boundary, which allows us to sample the critical boundary field efficiently, minimizing the effect of critical slowdown. To demonstrate this, we first generate one field configuration as shown in the left panel of Fig. 5. Then we push this field configuration back into the bulk field $\zeta$. We tweak the bulk field in the most IR degree of freedom by adding a small random Gaussian number. The variance of the added Gaussian variable is five times smaller than the variance of the bulk field degree of freedom. Under the EHM, we observe a global change of the boundary field configuration, as shown in Fig. 5. It is interesting to note that the change of the IR bulk field basically induces a global U(1) rotation of $\phi_{i}$ (see the insets of Fig. 5), which corresponds to the "Goldstone mode" associated with the "spontaneous symmetry breaking" in the fine-sized system, showing that the machine can identify the order parameter as the relevant IR degrees of freedom without prior knowledge about lowenergy modes of the system. We also check that the Hamiltonian Monte Carlo sampling in the bulk converges much faster compared with applying the same algorithm on the boundary (see Appendix D for more evidence). In connection to several recent works, our neural-RG architecture can be integrated to self-learning Monte Carlo approaches [48-54] to boost the numerical efficiency in simulating CFTs. The inverse RG transformation can also be used to generate super-resolution samples [55] for finite-size extrapolation of thermodynamic observables.

Now let us turn to the inference task. We can use the optimal EHM to push the boundary field back into the bulk and investigate the effective bulk theory $S_{\text {eff }}[\zeta]$ induced by the boundary CFT. As analyzed below Eq. (11), the mismatch between $P_{\text {post }}$ and $P_{\text {target }}$ will give rise to the residual correlation (mutual information) of the bulk-matter field, which can be used to probe the holographic bulk geometry. Assuming an emergent locality in the holographic bulk, the expectation is that the bulk effective theory $S_{\text {eff }}[\zeta]$ will take the following form in the continuum limit:

$$
S_{\mathrm{eff}}[\zeta]=\int_{\mathcal{M}} g^{\mu \nu} \partial_{\mu} \zeta^{*} \partial_{\nu} \zeta+m^{2}|\zeta|^{2}+u|\zeta|^{4}+\cdots,
$$

which describes the bulk field $\zeta$ on a curved space-time background $\mathcal{M}$ equipped with the metric tensor $g^{\mu \nu}$. Strictly speaking, $\zeta$ is not a single field but contains a tower of fields corresponding to different primary operators in the CFT. We choose to focus on the lightest component and model it by a scalar field, as it will dominate the bulk mutual information at large scale. Because the bulk field excitation is massive and cannot propagate far, we expect the mutual information between the bulk variables at two different points to decay exponentially with their geodesic distance in the bulk. Following this idea, suppose $\zeta_{i}=\zeta\left(x_{i}, z_{i}\right)$ and $\zeta_{j}=\zeta\left(x_{j}, z_{j}\right)$ are two bulk field variables, then their distance $d\left(\zeta_{i}: \zeta_{j}\right)$ can be inferred from their mutual information $I\left(\zeta_{i}: \zeta_{j}\right)$ as follows:

$$
d\left(\zeta_{i}: \zeta_{j}\right)=-\xi \ln \frac{I\left(\zeta_{i}: \zeta_{j}\right)}{I_{0}}
$$

where the correlation length $\xi$ and the information unit $I_{0}$ are global fitting parameters.

To estimate the mutual information among bulk field variables, we take a quadratic approximation of the bulk effective action $S_{\text {eff }}[\zeta] \simeq \sum_{i j} \zeta_{i}^{*} K_{i j} \zeta_{j}=\zeta^{\dagger} K \zeta$, ignoring the higherorder interactions of $\zeta$ for now. This amounts to relaxing the prior distribution of the bulk field $\zeta$ to a correlated Gaussian distribution

$$
P_{\text {prior }}^{\prime}[\zeta]=\frac{1}{\sqrt{\operatorname{det}\left(2 \pi K^{-1}\right)}} e^{-\zeta^{\dagger} K \zeta} .
$$


The kernel matrix $K$ is carefully designed to ensure positivity and bulk locality (see Appendix E for more details). To determine the best fit of $K$, we initiate the stage II training to learn the prior distribution with the EHM fixed at its optimal solution obtained in the stage I training, as illustrated in Fig. 2(c). We use the reparametrization trick [56] to sample the bulk field $\zeta$ from the correlated Gaussian in Eq. (15), then $\zeta$ is pushed to the boundary by the fixed EHM to evaluate the loss function $\mathcal{L}$ in Eq. (5), and the gradient signal can back-propagate to train the kernel $K$. As we relax the Gaussian kernel $K$ for training, we can see that the loss function will continue to drop in the stage II, as shown in Fig. 2(d). This indicates that the Gaussian model is learning to capture the residual bulk field correlation (at least partially), such that the overall performance of generation gets improved. One may wonder why we do not train the generative model $G$ and bulk field distribution $P_{\text {prior }}[\zeta]$ jointly. This is because there is a trade-off between these two objectives. For example, one can weaken the disentanglers in $G$ and push more correlation to the bulk field distribution $P_{\text {prior }}[\zeta]$. Such trade-off will undermine our objective of minimizing bulk mutual information in training a good EHM, therefore the two training stages should be separated, or at least assigned very different learning rates. Intuitively, the machine learns the background geometry in the stage I training and the bulk field theory (to the quadratic order) in the stage II training. The trade-off between the two training stages resembles the interchangeable roles between matter and space-time geometry in a gravity theory.

After the stage II training, we obtain the fitted kernel matrix $K$. The mutual information $I\left(\zeta_{i}: \zeta_{j}\right)$ can be evaluated from

$$
I\left(\zeta_{i}: \zeta_{j}\right)=-\frac{1}{2} \ln \left(1-\frac{\left\langle\zeta_{i}^{*} \zeta_{j}\right\rangle}{\left\langle\zeta_{i}^{*} \zeta_{i}\right\rangle\left\langle\zeta_{j}^{*} \zeta_{j}\right\rangle}\right)
$$

where the bulk correlation $\left\langle\zeta_{i}^{*} \zeta_{j}\right\rangle=\left(K^{-1}\right)_{i j}$ is simply given by the inverse of the kernel matrix $K$. Then we can measure the holographic distance $d\left(\zeta_{i}: \zeta_{j}\right)$ between any pair of bulk variables $\zeta_{i}$ and $\zeta_{j}$ following Eq. (14). To probe the bulk geometry, we further define the distance between two decimators $A$ and $B$ to be the average distance between all pairs of bulk variables separately associated with them,

$$
D(A: B)=\underset{\zeta_{i} \in A, \zeta_{j} \in B}{\operatorname{avg}} d\left(\zeta_{i}: \zeta_{j}\right) .
$$

The result is presented in Fig. 6(a). To visualize the bulk geometry qualitatively, we perform a multidimensional scaling to obtain a three-dimensional embedding of the decimators in Fig. 6(b). One can see that a hyperbolic geometry emerges in the bulk. To be more quantitative, we label each decimator by three coordinates $\left(x^{1}, x^{2}, z\right)$, where $x=\left(x^{1}, x^{2}\right)$ denotes its center position projected to the holographic boundary and $z=2^{l}$ is related to its layer depth $l$ (ascending from UV to IR). We found that the measured distance function follows the scaling behavior

$$
\begin{aligned}
& D\left(x^{1}, x^{2}, z: x^{1}+r, x^{2}, z\right) \propto \ln r, \\
& D\left(x^{1}, x^{2}, z: x^{1}, x^{2}, z+r\right) \propto r,
\end{aligned}
$$

as demonstrated in Figs. 6(c) and 6(d). These scaling behaviors agree with the geometry of a three-dimensional hyper-
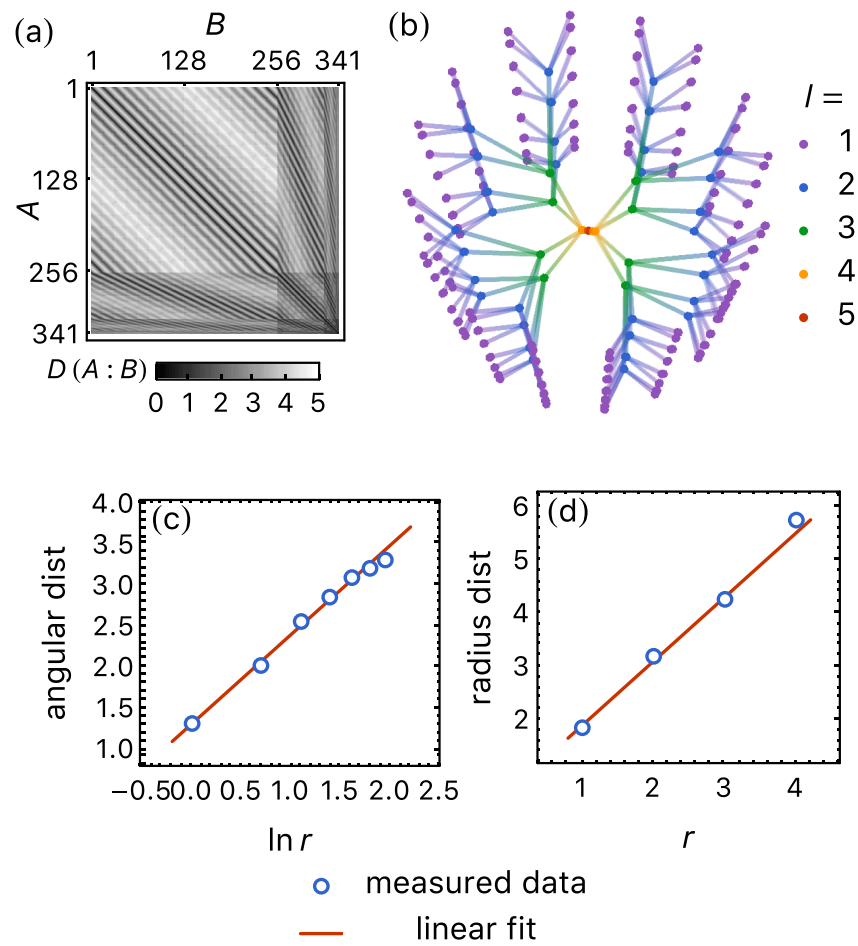

FIG. 6. (a) Distance matrix $D(A: B)$, indexed by the decimator indices $A, B$, obtained based on Eq. (17). (b) Visualization of the bulk geometry by multidimensional scaling projected to the leading three principle dimensions. Each point represents a decimator in the neural network, colored according to layers from UV to IR. The neighboring UV-IR links are add to guide the eye. Panels (c) and (d) show the distance scaling along (c) the angular and (d) the radius direction.

bolic space $H^{3}$, which corresponds to the $\mathrm{AdS}_{3}$ space-time under the Wick rotation of the time dimension. This indicates that the emergent bulk geometry is indeed hyperbolic at the classical level.

Our result demonstrates that the Luttinger liquid CFT can be approximately dual to a massive scalar fields on $\mathrm{AdS}_{3}$ background geometry. The duality is only approximate because we have assumed a classical geometry in the bulk, ignoring all the gravitational fluctuations. In $\mathrm{AdS}_{3} / \mathrm{CFT}_{2}$ correspondence, the bulk gravitational coupling $G_{N}=3 \ell / 2 c$ is inversely proportional to the central charge $c$ of the CFT [57]. The Luttinger liquid CFT has a relatively small central charge $c=1$ and hence a large gravitational coupling in the bulk, so we should not expect a classical dual description. It would be more appropriate to consider holographic CFTs which admit classical duals. However, our current method only applies to lattice field theories of bosons with explicit action functionals, which prevent us from studying interesting holographic CFTs. Generalizing the neural RG approach to involve fermions and gauge fields and to work with continuous space-time will be important directions for future development.

\section{SUMMARY AND DISCUSSIONS}

In conclusion, we introduced the neural RG algorithm to allow automated construction of EHM by machine learning 
instead of human design. Previously, the EHM was only designed for free fermion CFT. Using machine learning approaches, we are able to develop more general EHMs that also apply to interacting field theories. Given the QFT action as input, the machine effectively digests the information contained in the action and encodes it into the structure of the EHM network, which represents the emergent holographic geometry. Our result provides a concrete example that the holographic space-time geometry can emerge as the optimal generative network of a quantum field theory [58]. The obtained EHM simultaneously provides an information-preserving RG scheme and a generative model to reproduce the QFT, which could be useful for both inference and sampling tasks.

However, as a version of EHM, our approach also bares the limitations of EHM. By construction, the bulk geometry is discrete and classical, such that the model cannot resolve the sub-AdS geometry and cannot capture gravitational fluctuations. Recent development of neural ordinary differential equation approaches [59-61] are natural ways to extend our flow-based generative model to the continuum limit. Continuous formulation of real-space RG has been discussed in the context of gradient flows [62-64] and trivializing maps [65], where the RG flow equations are human-designed. Our research may pave the way for machine-learned RG flow equations for continuous holographic mappings. Our formalism also allows the inclusion of gravitational fluctuations in principle, by relaxing optimization to allow superposition of different EHMs. We conjecture that the bulk gravitational fluctuation could be partially captured by the fluctuation of neural network parameters. The learned EHM provides us a starting point to investigate the corrections on top of the classical geometry approximation, which may enable us to go beyond holographic CFTs and study the quantum gravity dual of generic QFTs. Another feature of EHM is that it is a oneto-one mapping of field configurations (operators) between bulk and boundary, while in holographic duality, a local bulk operator can be mapped to multiple boundary operators in different regions. A resolution $[66,67]$ of the paradox is that the nonunique bulk-boundary correspondence only applies to the low-energy freedoms in the bulk, which can be encoded on the boundary in a redundant and error-correcting manner. The bidirectional holographic code (BHC) [68,69] was proposed as an extension of the EHM to capture the error-correction property of the holographic mapping. Extending our current network design to realize machine-learned BHC will be another open question for future research.

\section{ACKNOWLEDGMENTS}

We acknowledge the stimulating discussions with XiaoLiang Qi, John McGreevy, Maciej Koch-Janusz, Cédric Bény, Koji Hashimoto, Wenbo Fu, and Shang Liu. S.-H.L and L.W. were supported by the National Natural Science Foundation of China under Grant No. 11774398 and the Strategic Priority Research Program of Chinese Academy of Sciences Grant No. XDB28000000. H.-Y.H. and Y.-Z.Y. are supported by a startup fund from UCSD. S.-H.L. and Y.-Z.Y. also benefit from the 2019 Swarma Club Study Camp supported by Kaifeng Foundation.

\section{APPENDIX A: MINIMAL BULK MUTUAL INFORMATION PRINCIPLE}

The maximal real-space mutual information (maxRMI) principle proposed in Refs. [30,38] aims to maximize the mutual information between the coarse-grained field and the fine-grained field in the surrounding environment at a single RG step. In this section, we show that the maxRMI principle can be derived from our minimal bulk mutual information (minBMI) principle under certain assumptions.

Let us set up the problem based on Fig. 7. Assuming $\phi_{A}$ and $\phi_{B}$ are field configurations in two neighboring regions $A$ and $B$ in the UV layer. Under one step of the RG transformation, $\phi_{A}$ gets mapped to the coarse-grained variable $\phi_{A}^{\prime}$ and the bulk variable $\zeta_{A}$, and the mapping is bijective. Similarly, another bijection takes $\phi_{B}$ to $\phi_{B}^{\prime}$ and $\zeta_{B}$. Eventually, $\phi_{A}^{\prime}$ and $\phi_{B}^{\prime}$ will be mapped to the bulk field $\zeta_{C}$ in deeper IR layers. Therefore, the random variables that appeared in Fig. 7 are related by the following bijections $f_{A}, f_{B}, f_{C}$ as

$\left(\phi_{A}^{\prime}, \zeta_{A}\right)=f_{A}\left(\phi_{A}\right), \quad\left(\phi_{B}^{\prime}, \zeta_{B}\right)=f_{B}\left(\phi_{B}\right), \quad \zeta_{C}=f_{C}\left(\phi_{A}^{\prime}, \phi_{B}^{\prime}\right)$

What are the information theoretical principles to guide the bijections $f_{A}, f_{B}, f_{C}$ toward good RG transformations? We propose the minBMI principle that these bijections should minimize the mutual information among the bulk variables,

$$
\min I\left(\zeta_{A}: \zeta_{B}\right)+I\left(\zeta_{A}: \zeta_{C}\right)+I\left(\zeta_{B}: \zeta_{C}\right)
$$

References [30,38] propose another principle, the maxRMI principle, whereby the RG transformation should maximize the mutual information between the coarse-grained variable (such as $\phi_{A}^{\prime}$ ) and its environments (such as $\phi_{B}$ ),

$$
\max I\left(\phi_{A}^{\prime}: \phi_{B}\right)
$$

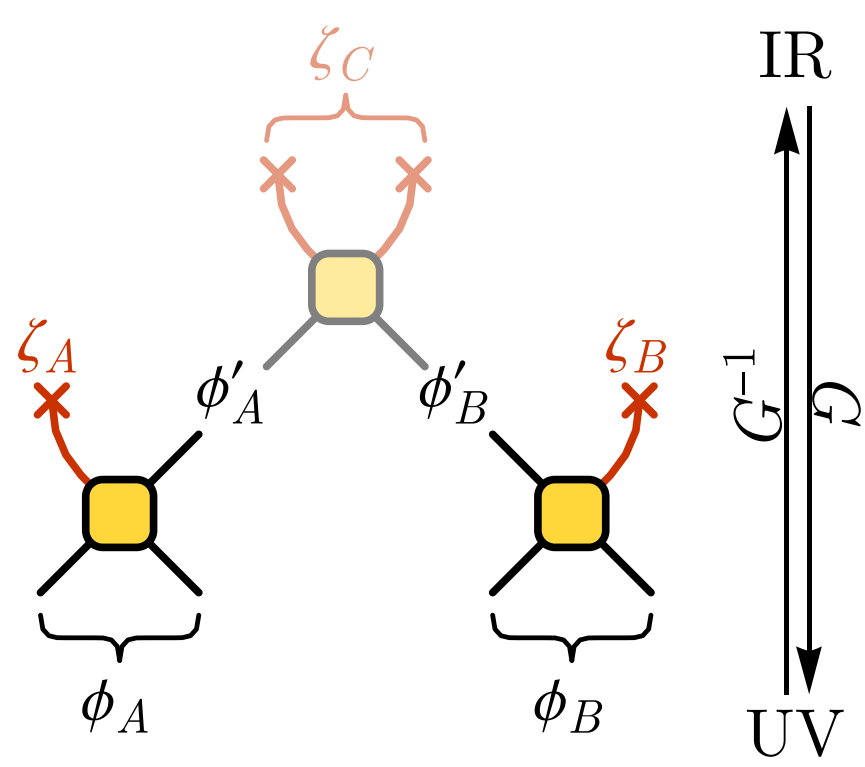

FIG. 7. Functional dependence of variables in the neural-RG network. Each block represents a bijective map. 
We can show that the objective of the maxRMI in Eq. (A3) is consistent with the objective of the minBMI in Eq. (A2) in the limit of UV-IR decoupling.

The minBMI principle aims to minimize mutual information among all bulk variables, both between different RG scales and within the same RG scale. Its objective has a broader scope than the maxRMI principle, because the latter does not specify its objectives across the RG scales. So to make a connection between these two principles, one must first restrict the scope of the minBMI principle to a single layer. This can be achieved by assuming that there is no mutual information between bulk variables at different RG scales. In our setup, this corresponds to $I\left(\zeta_{A}, \zeta_{B}: \zeta_{C}\right)=0$, which factorizes the joint probability $p\left(\zeta_{A}, \zeta_{B}, \zeta_{C}\right)=p\left(\zeta_{A}, \zeta_{B}\right) p\left(\zeta_{C}\right)$ and decouples the bulk variables between UV and IR. As a result, the mutual information between any bulk variables across different RG scales vanishes $I\left(\zeta_{A}: \zeta_{C}\right)=I\left(\zeta_{B}: \zeta_{C}\right)=$ 0 . This already minimizes the bulk mutual information across layers and reduces the minBMI objective in Eq. (A2) to

$$
\min I\left(\zeta_{A}: \zeta_{B}\right)
$$

In this UV-IR decoupled limit, we can prove that $\max I\left(\phi_{A}^{\prime}\right.$ : $\left.\phi_{B}\right)$ and $\min I\left(\zeta_{A}: \zeta_{B}\right)$ are equivalent.

The proof starts by considering the mutual information between $\phi_{A}$ and $\phi_{B}$. We can see that

$$
\begin{aligned}
I\left(\phi_{A}: \phi_{B}\right) & =I\left(\phi_{A}^{\prime}, \zeta_{A}: \phi_{B}\right) \\
& =I\left(\phi_{A}^{\prime}: \phi_{B}\right)+I\left(\zeta_{A}: \phi_{B}\right) \\
& =I\left(\phi_{A}^{\prime}: \phi_{B}\right)+I\left(\zeta_{A}: \phi_{B}^{\prime}, \zeta_{B}\right) \\
& =I\left(\phi_{A}^{\prime}: \phi_{B}\right)+I\left(\zeta_{A}: \phi_{B}^{\prime}\right)+I\left(\zeta_{A}: \zeta_{B}\right) \\
& =I\left(\phi_{A}^{\prime}: \phi_{B}\right)+I\left(\zeta_{A}: \zeta_{B}\right) .
\end{aligned}
$$

Here we have used the bijective property of $f_{A}, f_{B}, f_{C}$ to obtain $I\left(\phi_{A}: \phi_{B}\right)=I\left(\phi_{A}^{\prime}, \zeta_{A}: \phi_{B}\right), I\left(\zeta_{A}: \phi_{B}\right)=I\left(\zeta_{A}: \phi_{B}^{\prime}, \zeta_{B}\right)$, and $I\left(\zeta_{A}: \zeta_{C}\right)=I\left(\zeta_{A}: \phi_{A}^{\prime}, \phi_{B}^{\prime}\right)$. In the UV-IR decoupled limit, $I\left(\zeta_{A}: \zeta_{C}\right)=0$, so $I\left(\zeta_{A}: \phi_{A}^{\prime}, \phi_{B}^{\prime}\right)=0$, which further implies $I\left(\zeta_{A}: \phi_{A}^{\prime}\right)=I\left(\zeta_{A}: \phi_{B}^{\prime}\right)=0$. With these relations, all steps in Eq. (A5) are justified. On the left-hand side, $I\left(\phi_{A}: \phi_{B}\right)$ is determined by the field theory in the UV layer, which can be treated as a constant. For the given amount of information between regions $A$ and $B$, Eq. (A5) tells us that $I\left(\phi_{A}^{\prime}: \phi_{B}\right)$ and $I\left(\zeta_{A}: \zeta_{B}\right)$ are competing for information resources. Therefore maximizing $I\left(\phi_{A}^{\prime}: \phi_{B}\right)$ is equivalent to minimizing $I\left(\zeta_{A}: \zeta_{B}\right)$.

We can apply this argument layer by layer. Then to achieve the objective of the maxRMI principle, we need to minimize mutual information among bulk variables in the same RG scale, which is precisely the statement of the minBMI principle when restricted to each layer. In this sense, the maxRMI and minBMI principles are consistent. However, the minBMI principle actually relaxes the assumption that bulk variables at different RG scales are fully decoupled. Instead, we want to minimize mutual information among all bulk variables, including those across the scales. In this sense, the minBMI principle is more general than the maxRMI principle.

\section{APPENDIX B: DESIGN OF BIJECTORS}

We designed a set of symmetry-persevere bijectors to making sure that $U(1)$ symmetry of the boundary is preserved
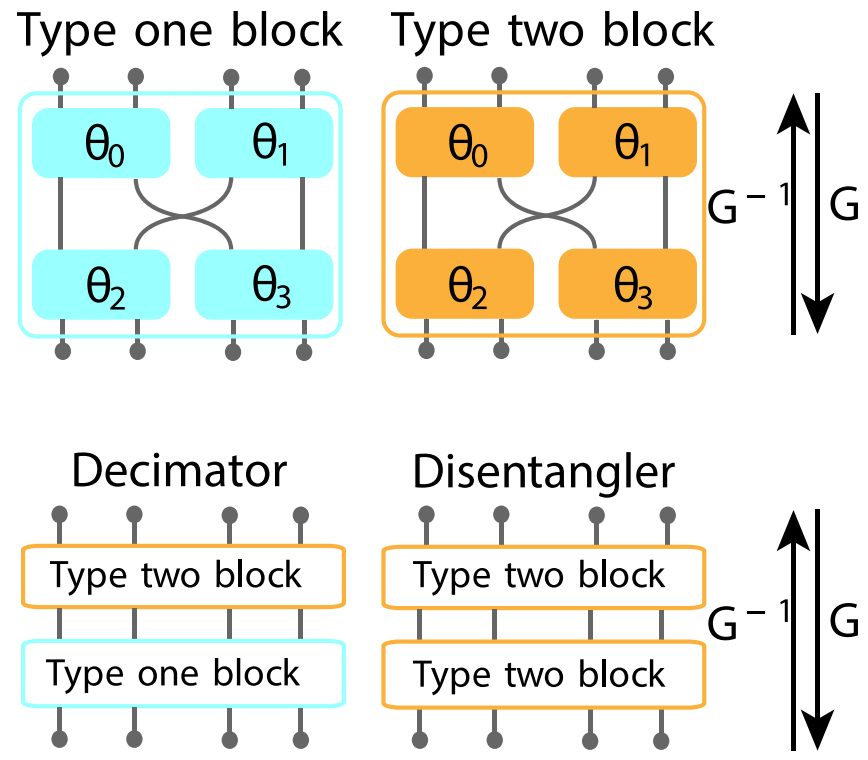

FIG. 8. Orthogonal transformation.

at each bijector. For the generative process, at each RG step, it takes the four complex degrees of freedom and they go through three layers of bijectors: $\mathcal{S}, \mathcal{O}$, and $\mathcal{A}$ :

(I) Scaling layer $(\mathcal{S})$ : At the scaling layer, each complex variables $\phi_{i}$ is multiplied by a factor $e^{\lambda_{i}}$. The inverse and the Jacobian of this transformation can be obtained easily.

(II) Orthogonal transformation layer $(\mathcal{O})$ : The orthogonal transformation in the disentangler and decimator is in general an $O(4)$ transformation. Instead, we implemented it by stacking multiple $O(2)$ transformations. In Fig. 8(a), each blue block represents the matrix:

$$
M_{\text {blue }}\left(\theta_{i}\right)=\left(\begin{array}{cc}
\sin \theta_{i} & \cos \theta_{i} \\
\cos \theta_{i} & -\sin \theta_{i}
\end{array}\right),
$$

and the orange block in Fig. 8(b) represents the matrix:

$$
M_{\text {orange }}\left(\theta_{i}\right)=\left(\begin{array}{cc}
\cos \theta_{i} & -\sin \theta_{i} \\
\sin \theta_{i} & \cos \theta_{i}
\end{array}\right) .
$$

$\theta_{i}$ in those blocks are training parameters. The arrangement of the type I and II blocks are so designed that when $M_{\text {blue }}\left(\theta_{i}=\right.$ $\pi / 4)$ and $M_{\text {orange }}\left(\theta_{i}=0\right)$ the network reproduces the ideal EHM originally proposed in Ref. [13]. We initialize the parameter to this ideal limit.

(III) Nonlinear layer $(\mathcal{A})$ For nonlinear part, we use the amplitude hyperbolic functions for complex field $\phi_{i}$. In teh coarse-graining direction, it acts in the following way:

$$
\begin{aligned}
& \operatorname{Re}(\zeta)=\sinh |\phi| \frac{\Re \phi}{|\phi|}, \\
& \operatorname{Im}(\zeta)=\sinh |\phi| \frac{\operatorname{Im} \phi}{|\phi|} .
\end{aligned}
$$

The corresponding inverse and Jacobian can be calculated easily. 


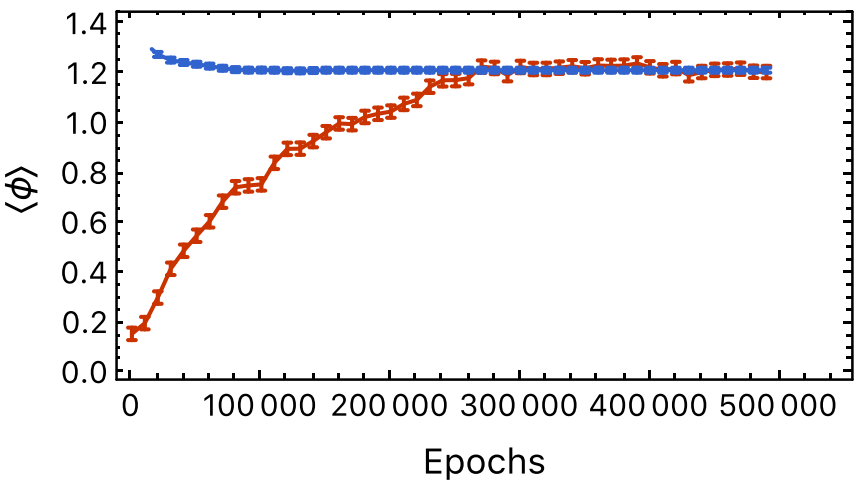

I Latent space HMC I Physical space HMC

FIG. 9. Hamiltonian Monte Carlo (HMC) simulations in the latent space and physical space.

\section{APPENDIX C: NEURAL NETWORK TRAINING}

All the training parameters of our neural RG network are contained in scaling bijectors and orthogonal transformation bijectors, as illustrated in Appendix B. We imposed translation invariance of our network at each layer, due to translation invariance of the system at each energy scale. The total number of training parameters scale with $O(\log (N))$, where $N$ is the size of boundary theory. The prefactor depends on the depth of bijector neural networks. In our case, the total number of training parameters are $24 \log _{2} N$. In order for faster convergence of the training, we first set learning rate for parameters contained in scaling bijectors as $10^{-2}$, and gradually reduce it to $10^{-4}$. And the learning rate for parameters contained in orthogonal transformation bijectors is always $10^{-4}$.

\section{APPENDIX D: MONTE CARLO SAMPLING EFFICIENCY}

We tested the numerical efficiency of our method by comparing the convergence rate between Hamiltonian Monte Carlo (HMC) on the boundary system, and HMC in the bulk system. Both methods are implemented by using TENSORFLOW probability API with same parameters. The result is shown in Fig. 9. As we can see, the HMC in the bulk system converges faster than the HMC on the boundary system.

\section{APPENDIX E: DESIGN OF THE CORRELATED GAUSSIAN PRIOR}

In finding the effective bulk field theory, we assume the bulk field is very massive. Under this assumption, higherorder interaction terms are irrelevant. Therefore, we use a correlated Gaussian distribution with positive definite kernel matrix $K$ as our effective bulk field theory. We also assumed locality of our effective bulk field theory, which means $K_{i j}$ is nonzero if and only if $\zeta_{i}$ and $\zeta_{j}$ are nearest neighbors in the bulk, including neighbors interscale and intrascale. To further reduce the fitting parameters of matrix $K$, we also imposed translation invariance of the bulk field at each scale. This is reasonable, because our RG scheme also has the translation invariance at each scale.

To ensure matrix $K$ is positive definite, we decomposed matrix $K$ into a set of positive semidefinite matrix and a mass term. Particularly,

$$
K=\sum_{\langle i j\rangle} \lambda_{i j}(|i\rangle\langle i|+| j\rangle\langle j|-| i\rangle\langle j|-| j\rangle\langle i|)+m \mathbb{I},
$$

where $\mathbb{I}$ is the identity matrix, and $\lambda_{i j}$ and $m$ are positive numbers. This ensures that the matrix $K$ we constructed is positive definite.
[1] E. Witten, Anti-de Sitter space and holography, Adv. Theor. Math. Phys. 2, 253 (1998).

[2] E. Witten, Anti-de Sitter space, thermal phase transition, and confinement in gauge theories, Adv. Theor. Math. Phys. 2, 505 (1998).

[3] S. S. Gubser, I. R. Klebanov, and A. M. Polyakov, Gauge theory correlators from non-critical string theory, Phys. Lett. B 428, 105 (1998).

[4] J. Maldacena, The large- $N$ limit of superconformal field theories and supergravity, Int. J. Theor. Phys. 38, 1113 (1999).

[5] J. de Boer, E. Verlinde, and H. Verlinde, On the holographic renormalization group, J. High Energy Phys. 8 (2000) 003.

[6] K. Skenderis, Lecture notes on holographic renormalization, Classical Quantum Gravity 19, 5849 (2002).

[7] I. Heemskerk and J. Polchinski, Holographic and Wilsonian renormalization groups, J. High Energy Phys. 06 (2011) 031.

[8] B. Swingle, Constructing holographic spacetimes using entanglement renormalization, arXiv:1209.3304.

[9] B. Swingle, Entanglement renormalization and holography, Phys. Rev. D 86, 065007 (2012).

[10] M. Nozaki, S. Ryu, and T. Takayanagi, Holographic geometry of entanglement renormalization in quantum field theories, J. High Energy Phys. 10 (2012) 193.
[11] V. Balasubramanian, M. Guica, and A. Lawrence, Holographic interpretations of the renormalization group, J. High Energy Phys. 01 (2013) 115

[12] L. P. Kadanoff, Scaling laws for Ising models near $T_{c}$, Physics (Long Island City, N. Y.) 2, 263 (1966).

[13] X.-L. Qi, Exact holographic mapping and emergent space-time geometry, arXiv:1309.6282.

[14] C. H. Lee and X.-L. Qi, Exact holographic mapping in free fermion systems, Phys. Rev. B 93, 035112 (2016).

[15] Y. Gu, C. H. Lee, X. Wen, G. Y. Cho, S. Ryu, and X.-L. Qi, Holographic duality between $(2+1)$-d quantum anomalous Hall state and $(3+1)$-d topological insulators, Phys. Rev. B 94, 125107 (2016).

[16] Y. LeCun, Y. Bengio, and G. Hinton, Deep learning, Nature (London) 521, 436 (2015).

[17] C. Bény, Deep learning and the renormalization group, arXiv:1301.3124.

[18] P. Mehta and D. J. Schwab, An exact mapping between the variational renormalization group and deep learning, arXiv: 1410.3831 .

[19] C. Bény and T. J. Osborne, The renormalization group via statistical inference, New J. Phys. 17, 083005 (2015).

[20] D. Oprisa and P. Toth, Criticality \& deep learning II: Momentum renormalisation group, arXiv:1705.11023. 
[21] H. W. Lin, M. Tegmark, and D. Rolnick, Why does deep and cheap learning work so well? J. Stat. Phys. 168, 1223 (2017).

[22] W.-C. Gan and F.-W. Shu, Holography as deep learning, Int. J. Mod. Phys. D 26, 1743020 (2017).

[23] G. Torlai and R. G. Melko, Learning thermodynamics with Boltzmann machines, Phys. Rev. B 94, 165134 (2016).

[24] L. Wang, Discovering phase transitions with unsupervised learning, Phys. Rev. B 94, 195105 (2016).

[25] E. P. L. van Nieuwenburg, Y.-H. Liu, and S. D. Huber, Learning phase transitions by confusion, Nat. Phys. 13, 435 (2017).

[26] G. Carleo and M. Troyer, Solving the quantum many-body problem with artificial neural networks, Science 355, 602 (2017).

[27] J. Carrasquilla and R. G. Melko, Machine learning phases of matter, Nat. Phys. 13, 431 (2017).

[28] G. Torlai, G. Mazzola, J. Carrasquilla, M. Troyer, R. Melko, and G. Carleo, Neural-network quantum state tomography, Nat. Phys. 14, 447 (2018).

[29] S.-H. Li and L. Wang, Neural Network Renormalization Group, Phys. Rev. Lett. 121, 260601 (2018).

[30] M. Koch-Janusz and Z. Ringel, Mutual information, neural networks and the renormalization group, Nat. Phys. 14, 578 (2018).

[31] Y.-Z. You, Z. Yang, and X.-L. Qi, Machine learning spatial geometry from entanglement features, Phys. Rev. B 97, 045153 (2018).

[32] K. Hashimoto, S. Sugishita, A. Tanaka, and A. Tomiya, Deep learning and the AdS/CFT correspondence, Phys. Rev. D 98, 046019 (2018).

[33] K. Hashimoto, S. Sugishita, A. Tanaka, and A. Tomiya, Deep learning and holographic QCD, Phys. Rev. D 98, 106014 (2018).

[34] Koji Hashimoto, AdS/CFT as a deep Boltzmann machine, Phys. Rev. D 99, 106017 (2019).

[35] L. Dinh, J. Sohl-Dickstein, and S. Bengio, Density estimation using Real NVP, arXiv:1605.08803.

[36] D. P. Kingma and P. Dhariwal, Glow: Generative flow with invertible $1 \times 1$ convolutions, arXiv:1807.03039.

[37] K. G. Wilson, The renormalization group and critical phenomena, Rev. Mod. Phys. 55, 583 (1983).

[38] P. M. Lenggenhager, Z. Ringel, S. D. Huber, and M. KochJanusz, Optimal Renormalization Group Transformation from Information Theory, Phys. Rev. X 10, 011037 (2020).

[39] M. Abadi, A. Agarwal, P. Barham, E. Brevdo, Z. Chen, C. Citro, G. S. Corrado, A. Davis, J. Dean, M. Devin, S. Ghemawat, I. Goodfellow, A. Harp, G. Irving, M. Isard, Y. Jia, R. Jozefowicz, L. Kaiser, M. Kudlur, J. Levenberg et al., TensorFlow: Largescale machine learning on heterogeneous systems (2015); software available from tensorflow.org.

[40] M. J. S. Beach, A. Golubeva, and R. G. Melko, Machine learning vortices at the Kosterlitz-Thouless transition, Phys. Rev. B 97, 045207 (2018).

[41] W. Zhang, J. Liu, and T.-C. Wei, Machine learning of phase transitions in the percolation and $X Y$ models, Phys. Rev. E 99, 032142 (2019).

[42] J. F. Rodriguez-Nieva and M. S. Scheurer, Identifying topological order via unsupervised machine learning, Nat. Phys. 15, 790 (2019).
[43] K. Zhou, G. Endrődi, L.-G. Pang, and H. Stöcker, Regressive and generative neural networks for scalar field theory, Phys. Rev. D 100, 011501 (2019).

[44] G. Vidal, Entanglement Renormalization, Phys. Rev. Lett. 99 , 220405 (2007).

[45] P. Olsson, Monte Carlo analysis of the two-dimensional $X Y$ model. II. Comparison with the Kosterlitz renormalizationgroup equations, Phys. Rev. B 52, 4526 (1995).

[46] M. Hasenbusch and K. Pinn, Computing the roughening transition of Ising and solid-on-solid models by BCSOS model matching, J. Phys. A: Math. Gen. 30, 63 (1997).

[47] M. Hasenbusch, The two-dimensional $X Y$ model at the transition temperature: A high-precision Monte Carlo study, J. Phys. A: Math. Gen. 38, 5869 (2005).

[48] J. Liu, H. Shen, Y. Qi, Z. Y. Meng, and L. Fu, Self-learning Monte Carlo method in fermion systems, Phys. Rev. B 95 , 241104 (2017).

[49] K.-I. Aoki and T. Kobayashi, Restricted Boltzmann machines for the long range Ising models, Mod. Phys. Lett. B 30, 1650401 (2016).

[50] L. Huang and L. Wang, Accelerated Monte Carlo simulations with restricted Boltzmann machines, Phys. Rev. B 95, 035105 (2017).

[51] J. Liu, Y. Qi, Z. Y. Meng, and L. Fu, Self-learning Monte Carlo method, Phys. Rev. B 95, 041101(R) (2017).

[52] Y. Nagai, H. Shen, Y. Qi, J. Liu, and L. Fu, Self-learning Monte Carlo method: Continuous-time algorithm, Phys. Rev. B 96, 161102(R) (2017).

[53] A. Tanaka and A. Tomiya, Towards reduction of autocorrelation in HMC by machine learning, arXiv:1712.03893.

[54] Y. Nagai, M. Okumura, and A. Tanaka, Self-learning Monte Carlo method with Behler-Parrinello neural networks, Phys. Rev. B 101, 115111 (2020).

[55] S. Efthymiou, M. J. S. Beach, and R. G. Melko, Super-resolving the Ising model with convolutional neural networks, Phys. Rev. B 99, 075113 (2019).

[56] D. P. Kingma and M. Welling, Auto-encoding variational Bayes, arXiv:1312.6114.

[57] J. D. Brown and M. Henneaux, Central charges in the canonical realization of asymptotic symmetries: An example from threedimensional gravity, Commun. Math. Phys. 104, 207 (1986).

[58] X. Dong and L. Zhou, Spacetime as the optimal generative network of quantum states: A roadmap to $\mathrm{QM}=\mathrm{GR}$ ? arXiv: 1804.07908

[59] R. T. Q. Chen, Y. Rubanova, J. Bettencourt, and D. Duvenaud, Neural ordinary differential equations, arXiv:1806.07366.

[60] L. Zhang, W. E, and L. Wang, Monge-Ampère flow for generative modeling, arXiv:1809.10188.

[61] W. Grathwohl, R. T. Q. Chen, J. Bettencourt, I. Sutskever, and D. Duvenaud, FFJORD: Free-form continuous dynamics for scalable reversible generative models, arXiv:1810.01367.

[62] K. Fujikawa, The gradient flow in $\lambda \phi^{4}$ theory, J. High Energy Phys. 03 (2016) 021.

[63] Y. Abe and M. Fukuma, Gradient flow and the renormalization group, Prog. Theor. Exp. Phys. 2018, 083 B02 (2018).

[64] A. Carosso, A. Hasenfratz, and E. T. Neil, Nonperturbative Renormalization of Operators in Near-Conformal Systems Using Gradient Flows, Phys. Rev. Lett. 121, 201601 (2018). 
[65] M. Lüscher, Trivializing maps, the Wilson flow and the HMC algorithm, Commun. Math. Phys. 293, 899 (2010).

[66] A. Almheiri, X. Dong, and D. Harlow, Bulk locality and quantum error correction in AdS/CFT, J. High Energy Phys. 04 (2015) 163.

[67] F. Pastawski, B. Yoshida, D. Harlow, and J. Preskill, Holographic quantum error-correcting codes: Toy models for the bulk/boundary correspondence, J. High Energy Phys. 06 (2015) 149.

[68] Z. Yang, P. Hayden, and X.-L. Qi, Bidirectional holographic codes and sub-AdS locality, J. High Energy Phys. 01 (2016) 175.

[69] X.-L. Qi, Z. Yang, and Yi-Zhuang You, Holographic coherent states from random tensor networks, J. High Energy Phys. 08 (2017) 060 . 